\title{
Injection of Onabotulinum Toxin A into the Bilateral External Oblique Muscle Attenuated Camptocormia: A Prospective Open-Label Study in Six Patients with Parkinson's Disease
}

\author{
Hiroyuki Todo · Hiroshi Yamasaki · Go Ogawa · Katsuya Nishida • \\ Naonobu Futamura · Itaru Funakawa
}

Received: June 19, 2018 / Published online: August 9, 2018

(C) The Author(s) 2018

\section{ABSTRACT}

Introduction: Camptocormia (severe bending of the spine) is a debilitating complication of Parkinson's disease (PD) without established treatment. Botulinum toxin (BT) may be beneficial, but data is scarce regarding the efficacy of administration of BT into the bilateral external oblique (EO) muscle for treatment of camptocormia in PD.

Methods: Six patients with PD and camptocormia, with flexion of the thoracic spine, were enrolled in the study. BT (75 or 90 units, onabotulinum toxin A) were injected into each EO bilaterally under sonographic guidance. Camptocormia angle (CA) was defined as the angle between the acromion-greater trochanter line and a vertical line. CA and disabling symptoms were evaluated during the treatment course.

Results: Two weeks after the injection of BT, the mean CA showed significant attenuation [median (interquartile range); $38^{\circ}\left(23.5^{\circ}\right)$ vs. $18^{\circ}$ $\left.\left(21^{\circ}\right), p=0.028\right]$. Subjective relief was present in

Enhanced digital content To view enhanced digital content for this article, go to https://doi.org/10.6084/ m9.figshare.6901976.

H. Todo $(\bowtie) \cdot$ H. Yamasaki · G. Ogawa · K. Nishida · N. Futamura · I. Funakawa Department of Neurology, National Hospital Organization Hyogo-Chuo National Hospital, 1314 Ohara, Sanda 669-1592, Japan e-mail: todo@hch.hosp.go.jp cases 1-3 and 6, and absent in cases 4 and 5 . Cases 1-3 received repeated injections to maintain the amelioration; in cases 1 and 2, this was for 1 year or longer, while falls of case 3 limited the amelioration.

Conclusion: Botulinum therapy into bilateral EO attenuated the angle of thoracic-level camptocormia in six patients with PD over the observation period of 2 weeks. The reproducibility of the results, long-term efficacy, and subjective relief of symptoms require further examination.

Keywords: Botulinum toxin; Camptocormia; External oblique muscle; Parkinson's disease

\section{INTRODUCTION}

Camptocormia is often associated with Parkinson's disease (PD) and is indicated by excessive spinal anteflexion, with exacerbation of symptoms when the patient is in an upright position and amelioration when in a recumbent position [1]. Long duration of PD, vertebral surgery, and dopamine agonists are representative cumulative factors leading to camptocormia [2]. The mechanisms of camptocormia are hypothesized to be dystonia, paravertebral myopathy, and disturbance of vertical perception; however, this has not been clarified [3]. Camptocormia is often refractory to treatment, including medication, physical therapy, and surgery $[3,4]$. 
As a treatment for camptocormia, local injection of botulinum toxin (BT) was first reported in 2005 in patients with parkinsonism and/or dystonia [5]. In the report, a single injection into the rectus abdominis (RA) muscle was effective for approximately 3 months. Following studies examined the efficacy of BT into the RA and iliopsoas muscles for camptocormia in PD; however, they failed to show significant improvement [6-8]. Using intramuscular injection of lidocaine, the external oblique (EO) muscle was first reported in 2012 as a therapeutic target for camptocormia in PD when the flexion point of the spine was between the lower thoracic and the upper lumbar level [9]. The same authors later showed that repeated lidocaine injection into the EO maintained improvement of camptocormia over 90 days of observation [10], and hyperactivity of the EO when tilting up could contribute to camptocormia by a dystonic mechanism [11]. In 2014, the first case of BT injection into unilateral EO and RA was reported [12]. In this report, a patient with PD received an injection of 200 units of onabotulinum toxin A into the right RA and left EO due to past excision of the left RA. The painful abdominal contraction, which was considered dystonia because of palpable abdominal contraction and hyperactivity in needle electromyography (nEMG), was ameliorated, as was the angle of camptocormia. Repeated injection of BT at intervals of 3-4 months successfully maintained the amelioration for 1 year or more. Apart from these reports, we found no published evidence of local injection into the EO for camptocormia in PD. Therefore, in this study we evaluated the therapeutic efficacy of BT injection into the bilateral EO muscle for camptocormia in multiple cases.

The aim of this study is an exploratory examination whether BT can attenuate the angle of camptocormia and disabling symptoms which are originated from camptocormia, and if any, whether clinical backgrounds (e.g. spinal degeneration) affect to the efficacy of BT.

\section{METHODS}

Six patients with PD and camptocormia (four females and two males, 54-79 years old,
$134-170 \mathrm{~cm}$ in height, $37.8-59.2 \mathrm{~kg}$ in weight) were enrolled in this prospective cohort study from June 2016 to February 2018. This study was approved by the local ethics committee of Hyogo-Chuo National Hospital and the patients provided written informed consent. This trial was registered in the Japan Medical Association Center for Clinical Trials Clinical Trial Registry (ID: JMA-IIA00371). Inclusion criteria were: meeting the UK Brain Bank criteria of PD [13], presence of debilitating symptoms or difficulty due to camptocormia, exacerbation of camptocormia in an upright position with amelioration in a recumbent position, and the flexion point in thoracic vertebrae. Exclusion criteria were: a family history of parkinsonism, presence of cognitive dysfunction (Mini-Mental State Examination score $\leq 25$ ), overlap of latero- or retro-flexion of the spine, and exhibiting diurnal or on/off fluctuation of camptocormia.

Camptocormia angle (CA) was defined as the angle between a vertical line and the line connecting the acromion with the greater trochanter, as defined in a prior study [2]. CA was evaluated in the lateral view on digital photographs taken at a distance of $2 \mathrm{~m}$ and a height of $1 \mathrm{~m}$, without any effort by the patients to correct their posture. We did not exclude patients with spinal lesions if the flexion angle of the spinal deformity was clearly milder than the observed CA. When the patient had painful abdominal contraction, the severity of the pain was evaluated using the visual analog scale (VAS) for painful abdominal contraction (range 0-100; 100 points being the worst) [14]. Objective stiffness of abdominal muscles $[5,12]$ was defined in this study as pathological contraction of abdominal muscles (including EO and RA) at inspection and/or palpation in a relaxed spine position. Muscle hyperactivity $(\mathrm{MH})$ in nEMG of abdominal muscles [12] was defined here as the presence of pathological involuntary discharges in a relaxed spine position. Tilttable EMG [11] was not available in our institution and thus, was not used.

Onabotulinum toxin-A (Botox ${ }^{\circledR}$; Allergan Plc, Dublin, Ireland) was injected into the bilateral EO muscle at a symmetrical dose under sonographic guidance. The injection site in each EO was the intersection point of the 
anterior axillary line and the transverse line through the umbilicus; therefore, each patient received two injections as a single treatment. Seventy-five (case 1) or 90 (cases 2-6) units of BT were injected into each EO (i.e. 150 or 180 units per patient) at a concentration of 100 units BT per $4 \mathrm{~mL}$ normal saline, since this dilution was our practice for the injection into large or wide muscles to gain widespread diffusion of BT. Using a previous report on cervical dystonia to help determine dosage, we limited the first dose to less than 180 units per patient [14]. Case 1 received a smaller dose of $\mathrm{BT}$ than the others because they were the first to receive BT in the EO in our institution.

For statistical analysis, Shapiro-Wilk testing was used to examine the normality of data distribution, and when the normality was not confirmed, the Wilcoxon signed-rank test was used to compare CA before and 2 weeks after the dose of BT by using analytic software, ExcelToukei Version 2018 (SSRI Co. Ltd., Tokyo, Japan). A $p$ value $<0.05$ obtained in a two-tailed test was considered as statistically significant. Long-term effects were sustained using maintenance doses of 100 units (case 1) or 120 units (cases 2-6) per EO, plus $30 \%$ of the original dose, in adequate intervals for each patient when the efficacy of BT had worn off. Antiparkinsonian medications were not changed during the observation period. All procedures performed in studies involving human participants were in accordance with the ethical standards of the institutional research committee and with the 1964 Helsinki Declaration and its later amendments or comparable ethical standards. Informed consent was obtained from all individual participants included in the study.

\section{RESULTS}

The profiles of the patients are shown in Table 1. The exacerbating factor of camptocormia was forward bending for the housework in case 2, and spinal compressive fracture or surgery in other cases. The most disabling symptoms relevant to camptocormia were painful abdominal contractions in cases 1 and
2 , dysbasia in case 3 , and difficulty in facing upward in cases 4-6. Except case 2, who had comorbid blepharospasm, none of the enrolled patients had dystonia or dystonia-associated pathologies other than camptocormia. Antiparkinsonian medications, including dopamine agonists (daily dose of pramipexole, $1.125 \mathrm{mg}$ in case 2 and $1.5 \mathrm{mg}$ in case 4 and 6; rotigotine, $27 \mathrm{mg}$ in case 1 and $4.5 \mathrm{mg}$ in case $3)$, were not changed during the observation in this study. Antiparkinsonian medications in the patients were unlikely to have induced or exacerbated camptocormia in daily examinations. The flexion point was medium (fifth-eighth) thoracic vertebrae in cases 1 and 5 and lower (ninth-twelth) thoracic vertebrae in the remaining cases. Objective stiffness of EO and $\mathrm{RA}$ and $\mathrm{MH}$ and/or continuous repetitive discharge (CRD) in nEMG were present in all cases; however, only cases 1 and 2 claimed pain due to abdominal contraction. CRD in EO has not been documented in any previous published reports. The patients had variable severity of spinal pathologies, and the deformity of the spine was severe in cases 4 and 6 , featured by lumbar herniation and lumbar canal stenosis. Atrophy of the paraspinal muscle in computed tomography was mild (i.e. can be explained by normal aging) in cases 1,2 , and 6 and moderate (i.e. considered as pathologic, but a certain amount of the muscle was remaining) in cases $3-5$. No adverse events were observed with the injection of BT. The efficacy of BT was noted, by subjective claim and/or objective inspection, 7-10 days after the injection. Two weeks after injection, CA was attenuated in all the six cases, as shown in Table 1 . The normality of the distribution of CA was not confirmed as significant $(p>0.05)$. The median (interquartile range) of CA showed significant attenuation $\left[38^{\circ}\left(23.5^{\circ}\right)\right.$ vs. $18^{\circ}\left(21^{\circ}\right), p=0.028$ ], comparing before and 2 weeks after the botulinum injection. Camptocormia-related symptoms were subjectively relieved in cases 1-3 and 6; however, cases 4 and 5 did not perceive the amelioration, hence cases 4 and 5 rejected the maintenance doses of BT. Patient 6 also declined the maintenance dose because he needed to care for his wife. The VAS of painful abdominal contraction in cases 1 and 2 improved from 84 and 98 to 4 and 77, 
Table 1 Clinical features of the patients

\begin{tabular}{|c|c|c|c|c|c|c|}
\hline Case & 1 & 2 & 3 & 4 & 5 & 6 \\
\hline Age (years) & 54 & 73 & 76 & 79 & 76 & 77 \\
\hline Sex & Male & Female & Female & Female & Female & Male \\
\hline $\begin{array}{l}\text { Hoehn \& Yahr } \\
\text { scale }\end{array}$ & 4 & 3 & 4 & 3 & 3 & 3 \\
\hline $\begin{array}{l}\text { PD duration } \\
\text { (years) }\end{array}$ & 18 & 16 & 6 & 10 & 5 & 9 \\
\hline $\begin{array}{l}\text { Camptocormia } \\
\text { duration (years) }\end{array}$ & 3 & 2 & 3 & 2 & 1 & 3 \\
\hline $\begin{array}{l}\text { Dopamine agonist } \\
(\mathrm{mg} / \text { day })\end{array}$ & RTG 27 & PPX 1.125 & RTG 4.5 & PPX 1.5 & Not used & PPX 1.5 \\
\hline $\begin{array}{l}\text { Exacerbating } \\
\text { factors of } \\
\text { camptocormia }\end{array}$ & $\begin{array}{l}\text { LH } \\
\text { surgery }\end{array}$ & Housework & SCF & SCF & SCF & LCS surgery \\
\hline Height $(\mathrm{cm})$ & 165 & 142 & 144 & 134 & 138 & 170 \\
\hline Body weight (kg) & 54.3 & 40.6 & 39.6 & 40.0 & 37.8 & 59.2 \\
\hline $\begin{array}{l}\text { Flexion point of } \\
\text { the spine }\end{array}$ & $\begin{array}{l}\text { Medium } \\
\text { Th }\end{array}$ & Lower $\mathrm{Th}$ & Lower $\mathrm{Th}$ & Lower Th & Medium Th & Lower Th \\
\hline $\begin{array}{l}\text { Spinal } x \text {-ray or } \\
\text { MRI }\end{array}$ & $\begin{array}{l}\text { LH Th11 } \\
\text { fracture }\end{array}$ & Mild LCS & $\begin{array}{l}\text { SC fracture, } \\
\text { mild LCS, } \\
\text { mild LH }\end{array}$ & $\begin{array}{l}\text { L2, and L3 } \\
\text { fracture, severe } \\
\text { LCS, severe LH }\end{array}$ & $\begin{array}{l}\text { L2 fracture, } \\
\text { moderate } \\
\text { LCS, mild } \\
\text { LH }\end{array}$ & $\begin{array}{c}\text { Th11 fracture, } \\
\text { severe LCS, } \\
\text { severe LH }\end{array}$ \\
\hline $\begin{array}{l}\text { Paraspinal muscle } \\
\text { atrophy }\end{array}$ & Mild & Mild & Moderate & Moderate & Moderate & Mild \\
\hline nEMG & $\begin{array}{c}\mathrm{MH} \text { in } \\
\mathrm{EO}\end{array}$ & $\begin{array}{l}\mathrm{MH} \text { in } \mathrm{EO}, \\
\mathrm{CRD} \text { in } \mathrm{EO} \\
\text { \& RA }\end{array}$ & $\mathrm{CRD}$ in $\mathrm{EO}$ & $\mathrm{CRD}$ in $\mathrm{EO} \& \mathrm{RA}$ & $\mathrm{MH}$ in $\mathrm{EO}$ & $\begin{array}{l}\mathrm{MH} \text { in } \mathrm{EO} \& \\
\mathrm{RA}\end{array}$ \\
\hline $\begin{array}{l}\text { Dose of BT (units } \\
\text { per EO) }\end{array}$ & 75 & 90 & 90 & 90 & 90 & 90 \\
\hline $\begin{array}{l}\text { CA (degrees, } \\
\text { before vs. } \\
2 \text { weeks after } \\
\text { BT) }\end{array}$ & 49 vs. 9 & 21 vs. 4 & 83 vs. 30 & 33 vs. 27 & 19 vs. 8 & 43 vs. 37 \\
\hline
\end{tabular}

$B T$ botulinum toxin, $C A$ camptocormia angle, $C R D$ continuous repetitive discharge, $C T$ computed tomography, EO external oblique, $L$ lumbar (L2 and L3 show the vertebrae with fracture), $L C S$ lumbar canal stenosis, $L S$ lumbar spondylosis, $L H$ lumbar herniation, $M H$ muscle hyperactivity, $M R I$ magnetic resonance imaging, $M U P$ motor unit potential, $n E M G$ needle electromyography, $P P X$ pramipexole, $P S M$ paraspinal muscle, $R A$ rectus abdominis, $R T G$ rotigotine, $S C$ sacrococcygeal, $S C F$ spinal compressive fracture, $T h$ thoracic vertebrae (Th11 shows the vertebrae with fracture) 
respectively. In case 3 , the time to walk a $10-\mathrm{m}$ straight line and their stride improved from $16.1 \mathrm{~s}$ with 29 steps, to $11.0 \mathrm{~s}$ with 22 steps after BT. Objective abdominal stiffness and nEMG findings persisted even after camptocormia was attenuated. Cases 1-3 received maintenance doses of 100 (case 1) or 120 (cases 2-3) units of BT per EO (i.e. 200 or 240 units per patient), and they obtained the same efficacy as the initial dose. By observing the decline of the effectiveness of each injection of BT, the interval of the maintenance dose was 4 months in case 1 , because the effectiveness of BT tapered off at 3 months. The therapeutic interval was 3 months in cases 2 and 3 because the effectiveness tapered off at 2 months. In the long term, cases 1 and 2 had good maintenance of amelioration of camptocormia for 1 year or more (CA 1 year after the BT injection was $9^{\circ}$ and $7^{\circ}$, and VAS of abdominal pain 1 year after was 21 and 74 in cases 1 and 2, respectively). However, several falls without spinal compressive fracture in case 3 re-exacerbated camptocormia, such that attenuation of CA remained suboptimal (CA at 1 year was $48^{\circ}$ ).

\section{DISCUSSION}

We observed attenuation of CA in six patients with PD who suffered from thoracic-level camptocormia, when comparing before treatment and 2 weeks after the botulinum injection into bilateral EO. Although the number of patients is small, this report is the first to show efficacy of BT in bilateral EO with statistically significant improvement in the angle of camptocormia; however, there are several limitations and points for discussion. Due to the study design of an open-label and non-blinded trial, the possibility of a placebo effect cannot be eliminated. However, the latency from the injection of BT to the emergence of efficacy (7-10 days) and duration of efficacy (2-3 months) was considered to be the effect of BT because this treatment effect curve is also generally observed with botulinum therapy $[15,16]$

In a previous report using lidocaine, consecutive injections were performed every
4-5 days due to the shortness of drug action (the effect of a single dose lasted for several days), to obtain efficacy in 90 days [10]. Both the previous report of BT into unilateral EO [12] and our results show that a single injection of BT had therapeutic efficacy for several months; however, a direct comparison between BT and lidocaine is needed for conclusive evidence of a difference in their effectiveness.

We used doses of 75-90 units per single EO, i.e. 150-180 units per patient, of onabotulinum toxin A as the initial dose, for the safety of our light-weight patients. Considering the doses of onabotulinum toxin A used in previous studies of 300-600 units per patient [5] and 200 units in unilateral EO [12], our doses may have been insufficient. However, given that the escalation of doses in the maintenance injections did not lead to further amelioration, our doses may have reached supramaximal levels. We selected a relatively low concentration of BT to obtain a broad spread because of the width of EO; however, actual spread of BT is difficult to determine and varies between individuals $[15,16]$. In addition, more exploration is needed to determine the optimal site of injection in the EO, and the best method of evaluating the CA.

Considering the individual variability in the therapeutic efficacy of BT, the fact that cases 4 and 6 had severe spinal degeneration and very limited efficacy for BT suggests that modification only in dystonia of the EO muscle is suboptimal to attenuate camptocormia comprehensively. As suggested in a previous report of surgical intervention for camptocormia, poor quality of the spine can limit the therapeutic effect [4]. Moreover, the maximal effect of BT in case 3 could not be maintained due to re-exacerbation of symptoms due to falls. Therefore, to modify exacerbating factors of camptocormia it may be necessary to provide long-term treatment. In terms of subjective relief of symptoms, cases 4 and 5 did not perceive the attenuation of the CA; therefore, CA alone may be an insufficient marker to evaluate amelioration of camptocormia. As case 3 achieved the ability to walk faster and with a larger stride, similar to a recent report that showed a relationship between camptocormia and gait [17], marginal symptoms/signs 
associated with camptocormia (i.e. painful contraction of abdominal muscles in cases 1 and 2 , and dysbasia in case 3 ) might need intensive examination for the correct evaluation of therapeutic efficacy. We expected objective stiffness of abdominal muscles, $\mathrm{MH}$ and/or CRD to act as biomarkers of camptocormia; however, this was not the case. We speculate that the cause of CRD is secondary muscle injury from excessive contraction due to dystonia, although CRD is a nonspecific sign of injury in motor neurons or muscles [18]. Other limitations of this study are heterogeneity in the duration of $\mathrm{PD}$, in the severity of $\mathrm{CA}$, in the exacerbating factors of camptocormia and other backgrounds of patients, the coexistence of spinal degeneration, dropout of cases 4-6 to examine the long-term effect of BT, and a smaller dose of BT in case 1 than the others.

Limitations are small sample size, open-label and non-blinded trial, heterogeneity of the backgrounds of patients, unexamined validity of the maneuver of botulinum injection (i.e. the injection site and dilution), not thorough evaluation of walking ability, and dropout of three patients in the long term.

\section{CONCLUSIONS}

To conclude, BT into the bilateral EO muscle attenuated the angle of thoracic-level camptocormia in six patients with PD over the observation period of 2 weeks. The reproducibility of results, long-term efficacy, and subjective relief of symptoms requires further examination.

\section{ACKNOWLEDGEMENTS}

The authors thank the participating patients for their involvement.

The authors disclosed the study design and results for the involved patients after the closure of this study, without violating the anonymity of the patients.

Funding. No funding or sponsorship was received for this study or publication of this article. The article processing charges were funded by the authors.

Authorship. All named authors meet the International Committee of Medical Journal Editors (ICMJE) criteria for authorship for this article, take responsibility for the integrity of the work as a whole, and have given their approval for this version to be published.

Author Contribution. HT developed the concept and design of the study and analyzed the data. HT, HY, NK, and IF performed data collection. HT and OG performed and evaluated nEMG. HT drafted and others critically revised the manuscript. NF evaluated radiologic features of the patients. IF approved the final manuscript.

Disclosures. Hiroyuki Todo, Hiroshi Yamasaki, Go Ogawa, Katsuya Nishida, Naonobu Futamura, and Itaru Funakawa have nothing to disclose.

Compliance with Ethics Guidelines. All procedures performed in studies involving human participants were in accordance with the ethical standards of the institutional research committee and with the 1964 Helsinki Declaration and its later amendments or comparable ethical standards. Informed consent was obtained from all individual participants included in the study.

Data Availability. All the data used in the analysis are presented in Table 1 . The datasets generated during and/or analyzed during the current study are not publicly available due to privacy protection of the participating patients, but are available from the corresponding author on reasonable request.

Open Access. This article is distributed under the terms of the Creative Commons Attribution-NonCommercial 4.0 International License (http://creativecommons.org/licenses/ by-nc/4.0/), which permits any noncommercial use, distribution, and reproduction in any medium, provided you give appropriate credit to the original author(s) and the source, provide 
a link to the Creative Commons license, and indicate if changes were made.

\section{REFERENCES}

1. Doherty KM, van de Warrenburg BP, Peralta MC, et al. Postural deformities in Parkinson's disease. Lancet Neurol. 2011;10:538-49.

2. Oeda T, Umemura A, Tomita S, Hayashi R, Kohsaka M, Sawada H. Clinical factors associated with abnormal postures in Parkinson's disease. PLoS One. 2013;8:e73547.

3. Margraf NG, Wrede A, Deuschl G, Schulz-Schaeffer WJ. Pathophysiological concepts and treatment of camptocormia. J Parkinsons Dis. 2016;6:485-501.

4. Galbusera F, Bassani T, Stucovitz E, et al. Surgical treatment of spinal disorders in Parkinson's disease. Eur Spine J. 2018;27:101-8.

5. Azher SN, Jankovic J. Camptocormia: pathogenesis, classification, and response to therapy. Neurology. 2005;65:355-9.

6. von Coelln R, Raible A, Gasser T, Asmus F. Ultrasound-guided injection into the iliopsoas muscle with botulinum toxin in camptocormia. Mov Disord. 2008;23:889-92.

7. Colosimo C, Salvatori FM. Injection of the iliopsoas muscle with botulinum toxin in camptocormia. Mov Disord. 2009;24:316-7.

8. Fietzek UM, Schroeteler FE, Ceballos-Baumann AO. Goal attainment after treatment of parkinsonian camptocormia with botulinum toxin. Mov Disord. 2009;24:2027-8.

9. Furusawa Y, Mukai Y, Kobayashi Y, Sakamoto T, Murata M. Role of the external oblique muscle in upper camptocormia for patients with Parkinson's disease. Mov Disord. 2012;27:802-3.
10. Furusawa Y, Mukai Y, Kawazoe T, et al. Long-term effect of repeated lidocaine injections into the external oblique for upper camptocormia in Parkinson's disease. Parkinsonism Relat Disord. 2013;19:350-4.

11. Furusawa Y, Hanakawa T, Mukai Y, et al. Mechanism of camptocormia in Parkinson's disease analyzed by tilt table-EMG recording. Parkinsonism Relat Disord. 2015;21:765-70.

12. Wijemanne S, Jimenez-Shahed J. Improvement in dystonic camptocormia following botulinum toxin injection to the external oblique muscle. Parkinsonism Relat Disord. 2014;20:1106-7.

13. Hughes AJ, Daniel SE, Kilford L, Lees AJ. Accuracy of clinical diagnosis of idiopathic Parkinson's disease: a clinico-pathological study of 100 cases. J Neurol Neurosurg Psychiatry. 1992;55:181-4.

14. Wu C, Xue F, Chang W, et al. Botulinum toxin type A with or without needle electromyographic guidance in patients with cervical dystonia. Springerplus. 2016;5:1292.

15. Ramirez-Castaneda J, Jankovic J, Comella C, Dashtipour K, Fernandez HH, Mari Z. Diffusion, spread, and migration of botulinum toxin. Mov Disord. 2013;28:1775-83.

16. Marsh WA, Monroe DM, Brin MF, Gallagher CJ. Systematic review and meta-analysis of the duration of clinical effect of onabotulinumtoxinA in cervical dystonia. BMC Neurol. 2014;14:91.

17. Tramonti C, Di Martino S, Unti E, et al. Gait dynamics in pisa syndrome and camptocormia: the role of stride length and hip kinematics. Gait Posture. 2017;57:130-5.

18. Fellows LK, Foster BJ, Chalk CH. Clinical significance of complex repetitive discharges: a case-control study. Muscle Nerve. 2003;28:504-7. 